\title{
TWO THEOREMS IN STABILITY THEORY ${ }^{1}$
}

\section{J. F. P. MARTIN}

In studies concerned with determining the stability of solutions of some systems of linear differential equations, it of ten is helpful to be able to transform the given system into another whose stability properties are known or are more easily obtainable. It is of interest, therefore, to consider transformations usable for this purpose, and to develop criteria to indicate when one system has the same stability properties as another. In Theorem 1 we give conditions sufficient to identify a Lyapunov transformation if certain properties are possessed by the coefficient matrix in the linear differential equation satisfied by the transformation, and in Theorem 2 we present an extension of Erugin's theorem [1, p. 122]. However, we need a few definitions and some preliminary work first.

Definition. A nonsingular matrix $Q=Q(t)$ is said to be a Lyapunov transformation if it is continuously differentiable for all $t \geqq 0$ and if $Q, Q^{-1}, Q^{\prime}$, and $\left(Q^{-1}\right)^{\prime}$ are each bounded. We designate the class of all such by the symbol $L(0, \infty)$. (Primes indicate differentiation.)

Definition. A matrix $X(t)$ is said to be reducible to a matrix $Y(t)$ if both are defined for all $t \geqq 0$ and if there exists a Lyapunov transformation such that $X=Q Y$ for all $t \geqq 0$. When this holds, clearly $Y$ is reducible to $X$ also.

Definition. Matrices $A(t)$ and $B(t)$ are said to be $L$-equivalent to each other if both are defined for $t \geqq 0$ and if there exists a Lyapunov transformation $Q=Q(t)$ such that $B=Q^{-1} A Q-Q^{-1} Q^{\prime}$ in which case it is easy to see that we also have $A=P^{-1} B P-P^{-1} P^{\prime}$ where $P=Q^{-1}$.

In defining the class $L(0, \infty)$, we follow Gantmacher [1] and Lyapunov [2] rather than Nemytskii and Stepanov [3]. Although $L(0, \infty)$ is a restricted class of transformations it is a very useful one, for Lyapunov (loc. cit.) showed that matrices in this class preserve the stability properties of the Zero solution of a linear system of differential equations. More precisely, if $X^{\prime}=A(t) X$ and $Y^{\prime}=B(t) Y$ and if $X=Q Y$ for some $Q$ in $L(0, \infty)$ then the Zero solution for $Y$ is asymptotically stable, stable, or unstable according as the Zero solution for $X$ is. Gantmacher $[1$, p. 117] points out that for fixed $n$,

Received by the editors August 9, 1965.

1 This material is taken from Part III of the author's dissertation (4) in partial fulfillment of the requirements for the degree of Doctor of Philosophy at the Stevens Institute of Technology, Hoboken, New Jersey. 
the $n \times n$ matrices in $L(0, \infty)$ form a group under multiplication, although we shall not make use of this concept. Of greater utility at the moment is the idea of an equivalence relation.

Lemma 1. Let an $n \times n$ matrix $A(t)$ be continuous for all $t \geqq 0$. Then the set $\{B(t)\}$ of all $n \times n$ continuous matrices $L$-equivalent to $A(t)$ form an equivalence class.

Proof. $A(t)$ is $L$-equivalent to itself under $I$, the identity matrix and we already have pointed out in the definition that if $A$ is $L$ equivalent to $B$ then $B$ is $L$-equivalent to $A$. From the equation $B=Q^{-1} A Q-Q^{-1} Q^{\prime}$ we see that for any $Q$ in $L(0, \infty), B$ is continuous if $A$ is. Finally, if $B=\left(P_{1}\right)^{-1} A P_{1}-\left(P_{1}\right)^{-1} P_{1}^{\prime}$ and if

$$
C=\left(P_{2}\right)^{-1} B P_{2}-\left(P_{2}\right)^{-1} P_{2}^{\prime},
$$

then

$$
\begin{aligned}
C & =\left(P_{2}\right)^{-1}\left(P_{1}\right)^{-1} A P_{1} P_{2}-\left(P_{2}\right)^{-1}\left(P_{1}\right)^{-1} P_{1}^{\prime} P_{2}-\left(P_{2}\right)^{-1} P_{2} \\
& =P_{3}^{-1} A P_{3}-P_{3}^{-1} P_{3}^{\prime}
\end{aligned}
$$

where $P_{3}=P_{1} P_{2}$ and clearly $P_{3}$ is in $L(0, \infty)$, so $A$ is $L$-equivalent to $C$. Q.E.D.

The almost obvious relationship between reducibility and $L$-equivalence is easy to show.

LEMma 2. Let $A(t)$ and $B(t)$ be continuous for $t \geqq 0$, and let $X(t)$ and $Y(t)$ satisfy: $X^{\prime}=A X, X(0)=C_{1}$, and $Y^{\prime}=B Y, Y(0)=C_{2}$, where $C_{1}$ and $C_{2}$ are any nonsingular constant matrices. Then $X$ is reducible to $Y$ if and only if $A$ and $B$ are L-equivalent.

Proof. If $X$ is reducible to $Y$, then $X=Q Y$ for some $Q$ in $L(0, \infty)$. Then $X^{\prime}=A Q Y=Q^{\prime} Y+Q Y^{\prime}$ whence $Y^{\prime}=\left(Q^{-1} A Q-Q^{-1} Q^{\prime}\right) Y=B Y$ so $B$ is $L$-equivalent to $A$.

Similarly, if $B=Q^{-1} A Q-Q^{-1} Q^{\prime}$ for some $Q$ in $L(0, \infty)$, then $Q^{\prime}=A Q-Q B$, and this is known to have the solution $Q=X Y^{-1}$ where $X^{\prime}=A X$ and $Y^{\prime}=B Y$. So we have $X=Q Y$ and $X$ is reducible to $Y$. Q.E.D.

\section{COROllary. Reducibility is an equivalence relation.}

We remark that it is possible to arrive at the same conclusions by using the group property mentioned earlier to show that reducibility is an equivalence relation and then investigating the relationship between the matrices $A(t)$ and $B(t)$. One of the advantages of the present approach, however, is that it tends to focus the emphasis on the 
coefficient matrices, which are the principal quantities in evidence when the solution is not yet known.

It is evident that every nonsingular constant matrix is a Lyapunov transformation and it is not difficult to construct others. However, as each Lyapunov transformation $Q(t)$ is continuously differentiable, it satisfies some linear system $Q^{\prime}=M(t) Q$ of differential equations with continuous coefficients. In Theorem 1 below, we give a set of conditions sufficient to insure that a fundamental solution matrix for a given system is a Lyapunov transformation. For the conditions stated in the theorem, it is well known that any fundamental solution matrix is bounded and has a bounded inverse, but the fact that it is a Lyapunov matrix does not seem to have been generally recognized. The proof of the theorem is essentially trivial, however, in that all that is required is a routine checking of the properties required of a Lyapunov transformation.

Theorem 1. Let $A(t)$ be continuous and bounded for $t \geqq 0$ and let $\int_{0}^{t}\|A(u)\| d u \leqq K<\infty$ for all $t \geqq 0$, where the norm of $A(t)$ is as defined below. Then any fundamental matrix $X(t)$ satisfying $X^{\prime}=A(t) X$ is a Lyapunov transformation.

Proof. We use the norm $\|A\|=\|A(t)\|=\max _{i}\left\{\sum_{j}\left|a_{i, j}(t)\right|\right\}$ for the operator $A(t)$. For any solution vector $x_{j}(t)$ in $x(t)$ we define $\left|x_{j}(t)\right|$ $=\left|x_{j}\right|(t)=\sum_{i}\left|x_{i}, j(t)\right|$ and $\|X(t)\|=\|X\|(t)=\max _{j}\left\{\left|x_{j}\right|: x_{j} \in X\right\}$. Now it is well known that $\left|X_{j}\right| \leqq\left|X_{j}(0)\right| \exp \int_{0}^{t}\|A(u)\| d u$ which is bounded, so $X(t)$ is bounded. Then as $A(t)$ is bounded and continuous it is clear from $\left\|X^{\prime}\right\| \leqq\|A(t)\| \cdot\|X\|$ that $X^{\prime}$ is also. The boundedness of the integral of the norm of $A$ implies that the trace of $A$ has a bounded integral also, so from $\operatorname{det} X=\operatorname{det} X(0) \exp \int_{0}^{t} \operatorname{tr} A(u) d u$ we see that $|\operatorname{det} X(t)|$ is bounded and is bounded away from zero. Hence the same is true of $\left|\operatorname{det} X^{-1}(t)\right|$.

$X(t)$ and $X^{-1}(t)$ are both continuously differentiable and bounded and $X^{-1}(t)$ satisfies the differential equation $Z^{\prime}=-Z A(t)$ so the transpose $\left(X^{-1}\right)^{T}$ satisfies $Y^{\prime}=-A^{T} Y$, and from $\left\|Y^{\prime}\right\| \leqq\left\|-A^{T}\right\|$ - $\|Y\|$ we see that $d X^{-1} / d t$ is bounded. Therefore $X(t)$ is in $L(0, \infty)$. Q.E.D.

It should be noted that the conditions stated in the preceding theorem are sufficient but not necessary to guarantee that a fundamental solution $X(t)$ is in $L(0, \infty)$ : For example, consider the onedimensional system: $x^{\prime}=(\sin t / t) x, x(0)=1$. The solution $x(t)=\exp$ $(S i(t))$ is a Lyapunov transformation although $\int_{0}^{t}|\sin u / u| d u$ is unbounded.

It would be desirable to have a set of necessary and sufficient con- 
ditions for the transformation of one system to another by a Lyapunov transformation, but the general problem here seems quite difficult. There are special situations, however, for which it is possible to give conditions sufficient to insure that two given systems are $L$-equivalent.

Theorem 2. Let $A(t)$ and $B(t)$ be continuous for $t \geqq 0$ and let $A(t)$ and $B(t)$ be L-equivalent to their Jordan canonical forms $J(A)=J(A)(t)$ and $J(B)=J(B)(t)$, respectively. Then $A(t)$ is L-equivalent to $B(t)$ if and only if for some ordering of the eigenvalues $a_{i}(t)$ of $A(t)$ and $b_{i}(t)$ of $B(t), J(A)-J(B)$ is a diagonal matrix for all $t \geqq 0$ and there exist constants $K_{1}$ and $K_{2}$ such that $\left|a_{i}(t)-b_{i}(t)\right| \leqq K_{2}$ and $\mid \int_{0}^{t} \operatorname{Re}\left(a_{i}(u)\right.$ $\left.-b_{i}(u)\right) d u \mid \leqq K_{1}$ for all $t \geqq 0$ and each $i=1,2, \cdots, n$.

Proof. Note first that $J(A)$ and $J(B)$ are continuous, for as $J(A)$ is $L$-equivalent to $A(t)$, there exists a $Q_{1}=Q_{1}(t)$ in $L(0, \infty)$ such that $J(A)=Q_{1}^{-1} A Q_{1}-Q_{1}^{-1} Q_{1}^{\prime}$ and each term on the right is continuous so $J(A)$ is also and the same argument applies as well to $J(B)$. It follows that the distribution of 1's on the first superdiagonal of each matrix does not change for any $t \geqq 0$.

We may assume that the eigenvalues of $J(A)$ and $J(B)$ are ordered at $t=0$ in any way desired, for if they are not, the desired ordering can be achieved in the following way. If $J(A)=Q_{1}^{-1} A Q_{1}-Q_{1}^{-1} Q_{1}^{\prime}$ then there exists a permutation matrix $P$ such that $P^{-1} J(A) P$ has the desired ordering at $t=0$, and

$$
P^{-1} J(A) P=\left(Q_{1} P\right)^{-1} A\left(Q_{1} P\right)-\left(Q_{1} P\right)^{-1} \frac{d}{d t}\left(Q_{1} P\right)
$$

so the desired ordering of $J(A)$ is $L$-equivalent to $A$ by the Lyapunov transformation $Q_{1} P$. The same procedure obviously applied to $J(B)$ as well.

Therefore, we can write:

$$
\begin{aligned}
& J(A)=J_{1}(A)+J_{2}(A)+\cdots+J_{k}(A), \\
& J(B)=J_{1}(B)+J_{2}(B)+\cdots+J_{m}(B) .
\end{aligned}
$$

Now by Lemma 1 it is necessary and sufficient to consider the $L$-equivalence of $J(A)$ and $J(B)$, and by Lemma 2 these are $L$-equivalent if and only if $X(t)$ is reducible to $Y(t)$, where $X(t)$ and $Y(t)$ are fundamental matrices satisfying $X^{\prime}=J(A) X$, and $Y^{\prime}=J(B) Y$.

Now assume that $J(A)$ is $L$-equivalent to $J(B)$. Then $X=Q Y$ for some $Q$ in $L(0, \infty)$, and $J(A)$ and $J(B)$ are continuous and each commutes with its integral so: 


$$
Q=X Y^{-1}=\exp \left(\int_{0}^{t} J(A)(u) d u\right) \exp \left(-\int_{0}^{t} J(B)(u) d u\right)
$$

All elements in $Q$ and $Q^{-1}$ are bounded for all $t \geqq 0$ and as $Q$ is an upper triangular matrix we see that the elements $q_{i, i}$ on the main diagonal of $Q$ are $q_{i, i}=\exp \left(\int_{0}^{t}\left(a_{i}(u)-b_{i}(u)\right) d u\right)$, so it is clear we must have $\left|\int_{0}^{t} \operatorname{Re}\left(a_{i}(u)-b_{i}(u)\right) d u\right| \leqq K_{1}$ for some positive real number $K_{1}$.

Now we show that the distribution of unit elements on the first superdiagonal must be the same for both $J(A)$ and $J(B)$. Let: $J(A)=D_{1}+E_{1}$ and $J(B)=D_{2}+E_{2}$, where $D_{1}$ and $D_{2}$ are the diagonal matrices whose elements are the eigenvalues $a_{i}$ of $A$ and $b_{i}$ of $B$, and $E_{1}$ and $E_{2}$ are continuous nilpotent matrices having all elements zero except perhaps for certain unit elements on the first superdiagonals. Then we can write:

$$
\begin{aligned}
X & =X(t)=\exp \int_{0}^{t}\left(D_{1}+E_{1}\right) d u \\
& =\left(\exp \int_{0}^{t} D_{1}(u) d u\right)\left(I+t E_{1}+\frac{t^{2} E_{1}{ }^{2}}{2 !}+\cdots+\frac{t^{n-1} E_{1}^{n-1}}{(n-1) !}\right) \\
& =p_{1}\left(t E_{1}\right) \exp \int_{0}^{t} D_{1}(u) d u, \\
Y^{-1} & =Y^{-1}(t)=\exp \int_{0}^{t}-\left(D_{2}+E_{2}\right) d u \\
& =\left(\exp -\int_{0}^{t} D_{2}(u) d u\right)\left(I-t E_{2}+t^{2} E_{2}^{2}-\cdots \pm \frac{t^{n-1} E_{2}^{n-1}}{(n-1) !}\right) \\
& =\left(\exp -\int_{0}^{t} D_{2}(u) d u\right) p_{2}\left(t E_{2}\right) .
\end{aligned}
$$

Then

$$
Q=p_{1}\left(t E_{1}\right)\left(\exp \int_{0}^{t}\left(D_{1}-D_{2}\right) d u\right) p_{2}\left(t E_{2}\right)
$$

and

$$
Q^{-1}=p_{2}\left(-t E_{2}\right)\left(\exp \int_{0}^{t}\left(D_{2}-D_{1}\right) d u\right) p_{1}\left(-t E_{1}\right)
$$

and both of these are continuous and bounded. But each element of $Q$ (and also of $Q^{-1}$ ) is a polynomial in $t$ with bounded coefficients 
which are also functions of $t$ in general. Hence, each $q_{i, j}$ in $Q$ can be bounded for all $t \geqq 0$ only if every coefficient of $t^{k}, k \geqq 1$, is zero in each polynomial so that $p_{1}\left(t E_{1}\right) p_{2}\left(t E_{2}\right)=I$. It follows that $J(A)$ and $J(B)$ have the same distribution of 1 's on their first superdiagonals so they commute for all $t \geqq 0$.

Consequently we now can write $Q$ as:

$$
Q=\exp \int_{0}^{t}(J(A)-J(B)) d u
$$

From the fact that $Q^{\prime}$ and $\left(Q^{-1}\right)^{\prime}$ are each bounded and $Q^{\prime}=$ $(J(A)-J(B)) \exp \int_{0}^{t}(J(A)-J(B)) d u$, it is easy to see that $J(A)-J(B)$ is a bounded diagonal matrix, so there exists a number $K_{2}$ such that $a_{i}(t)-b_{i}(t) \leqq K_{2}$ for all $t \geqq 0$. Now we have shown that if $A(t)$ and $B(t)$ are each $L$-equivalent to their Jordan canonical forms, and if these Jordan forms are $L$-equivalent to each other, then $A(t)$ and $B(t)$ must have the properties claimed.

Conversely, if $A(t)$ and $B(t)$ are each $L$-equivalent to their Jordan forms $J(A)$ and $J(B)$, and if they have the properties stated, then it is clear that $[J(A), J(B)]=0$, so with $X=\exp \int_{0}^{t} J(A) d u$ and $Y=\exp \int_{0}^{\imath} J(B) d u$ where $X^{\prime}=J(A) X$ and $Y^{\prime}=J(B) Y$ with $X(0)=\mathrm{I}$ $=Y(0)$ we put $Q=X Y^{-1}=\exp \int_{0}^{t}(J(A)-J(B)) d u$ and it is easy to see at once that $Q, Q^{\prime}, Q^{-1}$, and $\left(Q^{-1}\right)^{\prime}$ are continuous and bounded so that $Q$ is in $L(0, \infty)$ and $X=Q Y$. Consequently, $J(A)$ is $L$-equivalent to $J(B)$, and so $A(t)$ must also be $L$-equivalent to $B(t)$. Q.E.D.

Corollary 1 (ERugin's Theorem). If $A$ and $B$ are constant matrices then $A$ is L-equivalent to $B$ if and only if $J(A)$ and $J(B)$ have the same distribution of 1's on their first superdiagonals, and for corresponding eigenvalues $a_{i}$ of $A$ and $b_{i}$ of $B$ we have $\operatorname{Re}\left(a_{i}\right)=\operatorname{Re}\left(b_{i}\right)$ for $i=1,2, \cdots, n$.

It seems worthwhile also to mention as corollaries two additional facts emerging from the proof of the theorem.

Corollary 2. If $A(t)$ is continuous for $t \geqq 0$ and is L-equivalent to its Jordan canonical form $J(A)$, then $J(A)$ is continuous and hence the distribution of 1's on the first superdiagonal does not change at any $t \geqq 0$.

Corollary 3. A continuous nondiagonal Jordan canonical form $J(A)$ is not L-equivalent to any continuous diagonal Jordan canonical form $J(B)$.

We call attention to the fact that in Theorem 2 it is not required 
that $A(t)$ or $B(t)$ be bounded although the eigenvalues of $A(t)$ and $B(t)$ must be "sufficiently close" to each other. Consider the following example: Let

$$
A(t)=\frac{1}{t+1}\left[\begin{array}{cc}
t^{2}+t-1 & t+1 \\
-(t+1) & t^{2}+3 t+1
\end{array}\right] \quad \text { for } t \geqq 0
$$

and

$$
B(t)=\frac{1}{(t+1)^{2}}\left[\begin{array}{cc}
t^{3}+t^{2}-2 t-3 & 4(t+1)^{2} \\
-(t+1)^{2} & t^{3}+5 t^{2}+6 t+1
\end{array}\right] .
$$

Then by routine means we can find the eigenvalues and similarity transforms to find:

$$
\begin{aligned}
& J(A)=C_{1}^{-1} A C_{1}=\left[\begin{array}{cc}
t+\frac{t}{t+1} & 1 \\
0 & t+\frac{t}{t+1}
\end{array}\right], \\
& J(B)=C_{2}^{-1} B C_{2}=\left[\begin{array}{cc}
t+\frac{t^{2}+t-1}{(t+1)^{2}} & 1 \\
0 & t+\frac{t^{2}+t-1}{(t+1)^{2}}
\end{array}\right],
\end{aligned}
$$

where

$$
C_{1}=\left[\begin{array}{ll}
1 & 0 \\
1 & 1
\end{array}\right] \text { and } C_{2}=\left[\begin{array}{ll}
2 & 1 \\
1 & 1
\end{array}\right]
$$

then $a_{i}-b_{i}=1 /(t+1)^{2}$ and $\int_{0}^{t}\left(a_{i}-b_{i}\right) d u=(t-1) / 2(t+1)$ and both of these are bounded. So if we set:

$$
Q_{0}=\exp \left(\frac{t-1}{2(t+1)}\right) \cdot I .
$$

Then we find that $A$ is $L$-equivalent to $B$ under the Lyapunov transformation $Q=C_{2} Q_{0} C_{2}^{-1}$.

\section{BIBLIOGRAPHY}

1. F. R. Gantmacher, Teoriya matric, Gosudarstv. Izdat. Tehn.-Teor. Lt., Moscow, 1953; English transl., Vol. 2, Chelsea, New York, 1959.

2. A. M. Lyapunov, Problème général de la stabilité du mouvement, Annals of 
Mathematics Studies No. 17, Princeton Univ. Press, Princeton, N. J.; Oxford Univ. Press, London, 1947.

3. V. V. NemystkiY and V. V. Stepanov, Qualitative theory of differential equations, Princeton Univ. Press, Princeton, N. J., 1947.

4. J. F. P. Martin, On the exponential representation of solutions of systems of linear differential equations and an extension of Erugin's Theorem, Doctoral Dissertation, Stevens Institute of Technology, Hoboken, N. J., 1965.

Cornell Aeronautical laboratory, Inc., Buffalo 